\title{
Verbesserte Überlebensraten
}

Hier steht eine Anzeige.

Fragestellung: Verbessert die zusätzliche PCV-Chemotherapie (Procarbacin, Lomustin (CCNU), Vincristin) bei niedriggradigen Gliomen die Prognose gegenüber alleiniger Strahlentherapie?

Hintergrund: Die Bedeutung therapeutischer Interventionen bei Gliomen des WHO-Grads II bleibt umstritten. Patienten mit günstigen prognostischen Faktoren können zum Teil zunächst einfach beobachtet werden, während solche mit ungünstigen prognostischen Faktoren bezüglich progressionsfreien Überlebens von Strahlentherapie und vermutlich auch alkylierender Chemotherapie profitieren.

Patienten und Methodik: Die RTOG-Studie 9802 randomisierte 251 Patienten mit Astrozytomen, Oligoastrozytomen oder Oligodendrogliomen des damals gültigen WHO-Grads II (1998 bis 2002) in die Gruppen alleinige Strahlentherapie oder gefolgt von PCV-Chemotherapie. Die mediane Nachbeobachtungszeit betrug 11,9 Jahre, 55\% der Patienten waren zum Zeitpunkt der Publikation verstorben.

Ergebnisse: Das mediane Überleben mit der Kombinationstherapie war mit 13,3 gegenüber 7,8 Jahren deutlich höher (Hazard Ratio 0,59, $\mathrm{p}=0,003$ ). Das progressionsfreie Überleben nach zehn Jahren war mit $51 \%$ gegenüber $21 \%$ ebenfalls deutlich erhöht. Das Ergebnis war für alle drei Histologien vergleichbar.

Buckner JC, Shaw EG, Pugh SL et al. Radiation plus procarbazine, CCNU and vincristine in lowgrade glioma. N Engl J Med 2016; 374: $1344-55$
Schlussfolgerungen: Die Autoren folgern, dass die Kombination von PCV-Chemotherapie und Strahlentherapie den neuen Standard für nach gültigen Kriterien behandlungsbedürftige Patienten mit Gliomen des WHO-Grads II darstellen sollte.

\section{- Kommentar von Michael Weller, Zürich, Schweiz}

\section{Neuer Therapiestandard definiert}

Die RTOG-Studie 9802 liefert harte Daten für eine Intensivierung der Therapie in der Primärsituation bei Patienten mit behandlungsbedürftigen Gliomen des WHOGrads II. Zu beachten ist, dass viele Patienten im Kontrollarm, die initial nur bestrahlt wurden, zu einem späteren Zeitpunkt eine alkylierende Chemotherapie erhielten und dennoch bezüglich des Gesamtüberlebens einen erheblichen Nachteil erlitten. Wenngleich fortlaufend Bedenken gegenüber der langfristigen Verträglichkeit dieser Kombination geäußert werden und vermutet wird, dass das gleiche Resultat auch mit Temozolomid-Chemotherapie hätte erreicht werden können, muss konstatiert werden, dass hiermit für Patienten mit behandlungsbedürftigen WHO-Grad-II-Gliomen ein neuer Standard definiert ist.

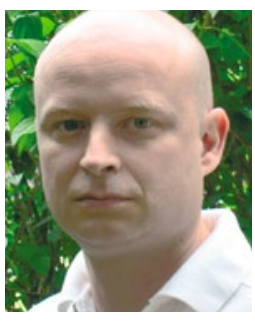

Prof. Dr. med. Michael Weller, Zürich/Schweiz 